INSTITUT NATIONAL DE RECHERCHE EN INFORMATIQUE ET EN AUTOMATIQUE

\title{
Sensitivity analysis of optimization problems under second order regular constraints
}

\author{
J. Frédéric Bonnans, Roberto Cominetti, Alexander Shapiro
}

\section{$\mathbf{N}^{\circ} 2989$}

Septembre 1996

THÈME 4 



\title{
RIN RIA
}

\section{Sensitivity analysis of optimization problems under second order regular constraints *}

\author{
J. Frédéric Bonnans ${ }^{\dagger}$, Roberto Cominetti ${ }^{\ddagger}$, Alexander Shapiro ${ }^{\S}$ \\ Thème 4 - Simulation et optimisation \\ de systèmes complexes \\ Projet PROMATH
}

Rapport de recherche $\mathrm{n}^{\circ} 2989$ - Septembre $1996-25$ pages

\begin{abstract}
We present a perturbation theory for finite dimensional optimization problems subject to abstract constraints satisfying a second order regularity condition. We derive Lipschitz and Hölder expansions of approximate optimal solutions, under a directional constraint qualification hypothesis and various second order sufficient conditions that take into account the curvature of the set defining the constraints of the problem. We also show how the theory applies to semi-definite optimization and, more generally, to semi-infinite programs in which the contact set is a smooth manifold and the quadratic growth condition in the constraint space holds. As a final application we provide a result on differentiability of metric projections in finite dimensional spaces.
\end{abstract}

Key-words: Sensitivity analysis, parametric optimization, optimal value function, directional constraint qualification, second order optimality conditions, semi-definite programming, semi-infinite programming, metric projection, directional differentiability.

(Résumé : tsvp)

\footnotetext{
* Research supported by the French-Chilean ECOS Program and by the European Community under contract 931091CL.

† INRIA, B.P. 105, 78153 Rocquencourt, France. Email: Frederic.Bonnans@inria.fr.

$\ddagger$ Universidad de Chile, Casilla 170/3 Correo 3, Santiago, Chile. Partially supported by Fondecyt 1961131. Email: rcominet@dim.uchile.cl.

$\S$ School of Industrial and Systems Engineering, Georgia Institute of Technology, Atlanta, Georgia 303320205, USA. Email: ashapiro@isye.gatech.edu.
}

Unité de recherche INRIA Rocquencourt

Domaine de Voluceau, Rocquencourt, BP 105, 78153 LE CHESNAY Cedex (France)

Téléphone : (33 1) 39635511 - Télécopie : (33 1) 39635330 


\section{Analyse de sensibilité de problèmes d'optimisation avec contraintes régulières au deuxième ordre}

Résumé : Nous présentons une théorie de la perturbation pour des problèmes d'optimisation en dimension finie satisfaisant une condition de régularité au deuxième ordre. Nous obtenons des développements de type Lipschitz et Hölder des solutions optimales approchées, sous l'hypothèse de qualification directionnelle ainsi que diverses conditions suffisantes du deuxième ordre qui prennent en compte la courbure de l'ensemble définissant les contraintes du problème. Nous exposons l'application de la théorie à l'optimisation semi définie et, plus généralement, à l'optimisation semi infinie quand l'ensemble de contact est une variété lisse et la condition de croissance quadratique dans l'espace des contraintes est vérifiée. L'application finale est un résultat sur la différentiabilité des projections orthogonales dans un espace de dimension finie.

Mots-clé : $\quad$ Analyse de sensibilité, optimisation paramétrique, coût optimal, qualification directionnelle, conditions d'optimalité du deuxième ordre, optimisation semi définie, optimisation semi infinie, projections orthogonales, différentiabilité directionnelle. 
AMS subject classifications 46N10, 47D20, 49K40, 58C15, 58C40, 90C31

\section{Introduction}

In this paper we present a theory that allows to compute the asymptotic expansions of the optimal value function $v(u)$, as well as the optimal or "nearly optimal" solutions $x(u)$ of parametric optimization problems of the form

$$
\operatorname{Min}_{x \in X} f(x, u) \text { subject to } G(x, u) \in K \text {. }
$$

While the theory is fairly complete in the framework of mathematical programming where the set $K$ is polyhedral, the question is far from being settled in general, particularly when dealing with infinite dimensional problems.

The differentiability properties of $v(u)$ and $x(u)$ strongly depend on the second order behavior of the unperturbed optimization problem, namely, quadratic growth and second order optimality conditions. Therefore, an essential difficulty in the general setting comes from the curvature (in a properly defined sense) that may appear with non-polyhedral constraint sets $K$. A number of generalized notions of polyhedricity have permitted to develop a perturbation theory for some relevant classes of infinite dimensional optimization problems $[3,13,18]$. Nevertheless, the curvature terms seem unavoidable for such problems as semi-infinite programming (i.e. minimization problems with an infinite number of inequality constraints) or semi-definite optimization. The latter is the particular case of $\left(P_{u}\right)$ where $Y=\mathcal{S}^{p}$ is the space of $p \times p$ symmetric matrices, and $K=\mathcal{S}_{+}^{p}$ is the cone of positive semi-definite matrices. When $f$ and $G$ are affine functions, the semi-definite optimization problem is known as the linear matrix inequalities (LMI) problem

$$
\operatorname{Min}_{x \in \mathbb{R}^{n}}\left\{c^{t} x: A_{0}+\sum_{i=1}^{n} x_{i} A_{i} \succeq 0\right\}
$$

where $A \succeq 0$ is used to denote $A \in \mathcal{S}_{+}^{p}$. This is an important particular case which has been recognized in the past few years as a very convenient framework for optimization problems arising in various fields $[7,8]$. However, relatively few papers have considered sensitivity analysis of such problems. The best results obtained so far, which are due to [26], provide explicit formulas for the expansion of the value function, solution and multiplier, under rather restrictive hypothesis which permit the use of the implicit function theorem.

An alternative approach for treating these difficult problems by taking into account the curvature of $K$, has emerged recently $[3,4,9,14,15]$. The novelty in this approach is the use of second order tangent sets and a second order property of the set $K$, called (inner) second order regularity, introduced in [3]. In [4] we discussed the weaker condition of outer second order regularity, under which there is no gap between second order necessary and sufficient optimality conditions. In the present paper we explore the implications and limitations of inner regularity in connection with sensitivity analysis of optimal solutions of perturbed optimization problems.

$\mathrm{RR} \quad \mathrm{n}^{\circ} 2989$ 
We begin by studying the sensitivity results in the abstract setting $\left(P_{u}\right)$. We then show how they apply in the framework of semi-infinite programming, and particularly for semidefinite optimization problems. Before proceeding with the abstract results, let us illustrate some of them through an elementary example where the computations can be carried out explicitly.

EXAMPle. Consider the family of convex semi-definite problems

$$
\operatorname{Min}_{x \in \mathbb{R}^{2}} x_{1}+\alpha x_{2}+\frac{1}{2} x_{2}^{2} \quad \text { s.t. } \quad\left(\begin{array}{ll}
x_{1} & u \\
u & x_{2}
\end{array}\right) \succeq 0,
$$

where $\alpha$ is a given nonnegative constant, and $u \in \mathbb{R}$ is a real parameter. When $u=0$, the above problem has a unique optimal solution $\bar{x}=(0,0)$, which satisfies Slater's condition. Moreover, there exists a unique Lagrange multiplier

$$
\bar{\lambda}=\left(\begin{array}{cc}
-1 & 0 \\
0 & -\alpha
\end{array}\right)
$$

and the second order growth condition (hence the strong second order sufficient condition) holds. The linearized problem, which captures the first order behavior of the optimal value function, is given by

$$
\underset{h}{\operatorname{Min}} h_{1}+\alpha h_{2} \text { s.t. } \quad\left(\begin{array}{ll}
h_{1} & 1 \\
1 & h_{2}
\end{array}\right) \succeq 0,
$$

with unique optimal solution $\bar{h}=(\sqrt{\alpha}, 1 / \sqrt{\alpha})$ if $\alpha>0$, and no solution if $\alpha=0$.

When $\alpha>0$ Theorem 4.1 (see section 4) applies: since the linearized problem has a unique optimal solution, the same holds for the auxiliary second order approximating problem and we get $x(u)=(\sqrt{\alpha}, 1 / \sqrt{\alpha}) u+o(u)$ for any $o\left(u^{2}\right)$-optimal trajectory $x(u), u \geq 0$.

When $\alpha=0$, Theorem 5.1 applies, leading to an expansion of the form $x(u)=o(\sqrt{u})$. We will discuss this case in section 7 .

Our perturbation analysis is based on: (i) a concept of second order regularity, (ii) second order sufficient optimality conditions, and (iii) the directional constraint qualification introduced in [3]. We distinguish between three basic cases, in the spirit of [3,5]. The first and second cases, illustrated in the example above, are when the strong and weak (second order) sufficient optimality conditions hold, leading respectively to Lipschitz and Hölder stability of optimal trajectories. The third case is when the set of Lagrange multipliers is empty, and the optimal solutions are once again Hölder stable of degree $1 / 2$. The derived results are similar to those obtained for nonlinear programming problems, except for the additional terms related to the curvature of the set $K$.

The paper follows the method of upper and lower estimates of the objective function $[3,5,12,14,22,23]$. The upper estimates are those obtained in [3]. The novelty lies in the theory of lower estimates and the expansion of approximate solutions. 


\section{Preliminaries}

In this section we review some known results on first order sensitivity analysis and second order optimality conditions, that are needed in the sequel. We also fix the notations used throughout the paper.

\subsection{Basic notation}

We consider the parametrized family of optimization problems $\left(P_{u}\right)$ with $f: X \times U \rightarrow \mathbb{R}$ and $G: X \times U \rightarrow Y$ mappings of class $\mathcal{C}^{2}$. The space of "decisions" $X$ is assumed to be finite dimensional, $U$ is a topological vector space of "parameters", and the "constraint" set

$K$ is a nonempty closed convex subset of the Banach space $Y$ with topological dual $Y^{*}$. The feasible set, optimal value, and set of optimal solutions of $\left(P_{u}\right)$ are denoted respectively

$$
\begin{aligned}
\Phi(u) & :=\{x \in X: G(x, u) \in K\}, \\
v(u) & :=\inf \{f(x, u): x \in \Phi(u)\}, \\
S(u) & :=\operatorname{Argmin}\{f(x, u): x \in \Phi(u)\} .
\end{aligned}
$$

Similarly, given an optimization problem $(P)$, we denote by $\Phi(P), v(P)$ and $S(P)$, the feasible set, the optimal value, and the set of optimal solutions of $(P)$.

For $u=0$ we view the corresponding optimization problem $\left(P_{0}\right)$ as unperturbed and assume that it has an optimal solution $x_{0} \in S(0)$. We shall consider perturbations along a fixed direction $d \in U$, that is to say, we investigate the local behavior of the optimal value and optimal solutions of the problems $\left(P_{t d}\right)$ for small $t \geq 0$. For $\varepsilon \geq 0$, we say that a point $x \in X$ is an $\varepsilon$-optimal solution of $\left(P_{u}\right)$ if $x \in \Phi(u)$ and $f(x, u) \leq v(u)+\varepsilon$. For a nonnegative valued function $\varepsilon(t)$ we shall also consider trajectories $x(t)$ of $\varepsilon(t)$-optimal solutions of $\left(P_{t d}\right)$.

The support function of $T \subset Y$ at $y^{*} \in Y^{*}$ is $\sigma\left(y^{*}, T\right):=\sup \left\{\left\langle y^{*}, y\right\rangle: y \in T\right\}$, while $\operatorname{dist}(y, T):=\inf \{\|y-z\|: z \in T\}$ is the distance function to $T$. The tangent and normal cones to the set $K$ at the point $y \in K$ are defined as

$$
\begin{aligned}
T_{K}(y) & :=\{h \in Y: \operatorname{dist}(y+t h, K)=o(t)\} \\
N_{K}(y) & :=\left\{y^{*} \in Y^{*}:\left\langle y^{*}, h\right\rangle \leq 0, \forall h \in T_{K}(y)\right\} .
\end{aligned}
$$

Finally, for a function $h: Y \rightarrow \mathbb{R}$, we denote by $h^{\prime}(y, d)$ its directional derivative

$$
h^{\prime}(y, d)=\lim _{t \downarrow 0} \frac{h(y+t d)-h(y)}{t} .
$$

If $h^{\prime}(y, d)$ exists for every $d \in Y$ we say that $h$ is directionally differentiable at $y$, and we define (when it exists) the parabolic second order directional derivative

$$
h^{\prime \prime}(y ; d, w)=\lim _{t \downarrow 0} \frac{h\left(y+t d+\frac{1}{2} t^{2} w\right)-h(y)-t h^{\prime}(y, d)}{\frac{1}{2} t^{2}} .
$$




\section{$2.2 \quad$ First order analysis}

Optimality conditions for $\left(P_{u}\right)$ are usually stated in terms of the associated Lagrangian and generalized Lagrangian

$$
\begin{aligned}
L(x, \lambda, u) & :=f(x, u)+\langle\lambda, G(x, u)\rangle, \quad \lambda \in Y^{*}, \\
L^{g}(x, \alpha, \lambda, u) & :=\alpha f(x, u)+\langle\lambda, G(x, u)\rangle, \quad(\alpha, \lambda) \in \mathbb{R} \times Y^{*} .
\end{aligned}
$$

The Fritz John necessary conditions for a local minimum $x$ of $\left(P_{u}\right)$ are

$$
D_{x} L^{g}(x, \alpha, \lambda, u)=0, \alpha \geq 0, \lambda \in N_{K}(G(x, u)) .
$$

The set $\Lambda_{u}^{g}(x)$ of generalized Lagrange multipliers $(\alpha, \lambda) \neq(0,0)$ satisfying (2.3), may be empty when $Y$ is a general Banach space. However $\Lambda_{u}^{g}(x) \neq \emptyset$ in two important cases: when $Y$ is finite dimensional and also when $K$ has a non empty interior $[17,28]$.

If the multiplier $\alpha$ in (2.3) is non zero, then we can take $\alpha=1$ and the corresponding first order necessary conditions become

$$
D_{x} L(x, \lambda, u)=0, \lambda \in N_{K}(G(x, u)) .
$$

The set $\Lambda_{u}(x)$ of Lagrange multipliers satisfying $(2.4)$ is non empty and bounded $[19,28]$, whenever the following constraint qualification, due to Robinson [20], holds

$$
0 \in \operatorname{int}\left\{G(x, u)+D_{x} G(x, u) X-K\right\} .
$$

Let us summarize the first order differentiability properties of the optimal value function $v(u)$ and the optimal solutions. To this end, for the given perturbation direction $d \in U$ and the (unperturbed) optimal solution $x_{0} \in S(0)$, we consider the following linearization of the family of problems $\left(P_{t d}\right)$,

$$
\operatorname{Min}_{h} D f\left(x_{0}, 0\right)(h, d) \text { s.t. } D G\left(x_{0}, 0\right)(h, d) \in T_{K}\left(G\left(x_{0}, 0\right)\right),
$$

together with its dual (cf. $[1,27])$

$$
\operatorname{Max}_{\lambda \in \Lambda_{0}\left(x_{0}\right)} D_{u} L\left(x_{0}, \lambda, 0\right) d .
$$

The directional constraint qualification at the point $x_{0}$ in the direction $d$, which is essentially Robinson's constraint qualification for $\left(P L_{d}\right)$, is (cf. [1] and [3, Part I])

$$
0 \in \operatorname{int}\left\{G\left(x_{0}, 0\right)+D G\left(x_{0}, 0\right)\left(X \times \mathbb{R}_{+} d\right)-K\right\} .
$$

It is clear that $(C Q)$ implies $(D C Q)$, and that both conditions are equivalent if $d=0$. 
Theorem 2.1 Suppose that (DCQ) holds. Then there is no duality gap between problems $\left(P L_{d}\right)$ and $\left(D L_{d}\right)$, i.e. $v\left(P L_{d}\right)=v\left(D L_{d}\right)$, and

$$
\limsup _{t \downarrow 0} \frac{v(t d)-v(0)}{t} \leq v\left(P L_{d}\right)
$$

Moreover, the common value $v\left(P L_{d}\right)=v\left(D L_{d}\right)$ is finite if and only if $\Lambda_{0}\left(x_{0}\right) \neq \emptyset$, in which case the set $S\left(D L_{d}\right)$ of optimal solutions of $\left(D L_{d}\right)$, is a non empty weak* compact subset of $\Lambda_{0}\left(x_{0}\right)$.

Furthermore, if there exists an o(t)-optimal trajectory $\bar{x}(t)$ of $\left(P_{t d}\right)$ such that $\left\|\bar{x}(t)-x_{0}\right\|=$ $O(t)$, then the directional derivative $v^{\prime}(0, d)$ exists and $v^{\prime}(0, d)=v\left(D L_{d}\right)$. In the latter case $S\left(P L_{d}\right)$ coincides with the set of accumulation points of the differential quotients $\left(x(t)-x_{0}\right) / t$ where $x(t)$ ranges over the set of all possible o $(t)$-optimal trajectories of $\left(P_{t d}\right)$.

For a discussion of this result and relevant references, the reader is referred to [6].

\subsection{Second order optimality conditions}

Let us briefly state the theory of second order optimality conditions for the unperturbed problem $\left(P_{0}\right)$. To this end we skip the argument $u$, and all derivatives are understood with respect to $x$ only.

Recall that the second order tangent set to $K$ at the point $y \in K$ in the direction $z \in T_{K}(y)$ is defined as

$$
T_{K}^{2}(y, z):=\left\{w \in Y: \operatorname{dist}\left(y+t z+\frac{1}{2} t^{2} w, K\right)=o\left(t^{2}\right), t \geq 0\right\},
$$

and that (under some first order qualification conditions) a second order necessary optimality condition is given by (see $[4,9,15]$ )

$$
\sup _{(\alpha, \lambda) \in \Lambda^{g}\left(x_{0}\right)}\left\{D_{x x}^{2} L^{g}\left(x_{0}, \alpha, \lambda\right)(h, h)-\sigma\left(\lambda, \mathcal{T}_{K}^{2}(h)\right)\right\} \geq 0, \quad \forall h \in C\left(x_{0}\right),
$$

where $\mathcal{T}_{K}^{2}(h):=T_{K}^{2}\left(G\left(x_{0}\right), D G\left(x_{0}\right) h\right)$, and $C\left(x_{0}\right)$ is the critical cone

$$
C\left(x_{0}\right):=\left\{h \in X: D f\left(x_{0}\right) h \leq 0 ; D G\left(x_{0}\right) h \in T_{K}\left(G\left(x_{0}\right)\right)\right\} .
$$

Unfortunately, for sufficient conditions it is not enough to change the weak inequality in (2.7) into a strict one, but one needs the following concept (see [4]),

Definition 2.1 $A$ set $\mathcal{A} \subset Y$ is an upper second order approximation set for $K$ at the point $y \in K$ in the direction $z \in T_{K}(y)$ with respect to a linear mapping $M: X \rightarrow Y$, if for any sequence $y+t_{k} z+\frac{1}{2} t_{k}^{2} r_{k} \in K$, where $t_{k} \downarrow 0$ and $r_{k}=M q_{k}+a_{k}$ with $\left\{a_{k}\right\}$ being a convergent sequence in $Y$ and $\left\{q_{k}\right\} \subset X$ satisfying $t_{k} q_{k} \rightarrow 0$, the following condition holds

$$
\lim _{k \rightarrow \infty} \operatorname{dist}\left(r_{k}, \mathcal{A}\right)=0
$$

$\mathrm{RR} \mathrm{n}^{\circ} 2989$ 
If this holds for any $M$, i.e. (2.8) is satisfied for any sequence $y+t_{k} z+\frac{1}{2} t_{k}^{2} r_{k} \in K$ such that $t_{k} \downarrow 0$ and $t_{k} r_{k} \rightarrow 0$, we say that $\mathcal{A}$ is an upper second order approximation set for $K$ at $y$ in the direction $z$.

A sufficient condition for $x_{0}$ to be a local optimum is (see [4])

$$
\sup _{(\alpha, \lambda) \in \Lambda^{g}\left(x_{0}\right)}\left\{D_{x x}^{2} L^{g}\left(x_{0}, \alpha, \lambda\right)(h, h)-\sigma(\lambda, \mathcal{A}(h))\right\}>0, \forall h \in C\left(x_{0}\right) \backslash\{0\},
$$

where $\mathcal{A}(h)$ is any upper second order approximation set for $K$ at the point $G\left(x_{0}\right)$ in the direction $D G\left(x_{0}\right) h$ with respect to the linear mapping $D G\left(x_{0}\right): X \rightarrow Y$. As a matter of fact, (2.9) implies the following quadratic growth condition at $x_{0}$ : there exist a constant $c>0$ and a neighborhood $N$ of $x_{0}$ such that

$$
f(x) \geq f\left(x_{0}\right)+c\left\|x-x_{0}\right\|^{2}, \quad \forall x \in \Phi\left(P_{0}\right) \cap N .
$$

Notice that (2.9) requires the set $\Lambda^{g}\left(x_{0}\right)$ to be nonempty, i.e. it implies that the corresponding (Fritz John) first order necessary conditions hold at $x_{0}$. We refer to (2.9) as the generalized second order sufficient condition, and to the term $\sigma(\lambda, \mathcal{A}(h))$ appearing in $(2.9)$ as the sigma term.

Since $T_{K}^{2}(y, z)$ is contained in every upper second order approximation set $\mathcal{A}$, the gap between (2.7) and (2.9) reduces to a change from weak to strict inequality whenever one can take $\mathcal{A}(h)=\mathcal{T}_{K}^{2}(h)$. This remark leads to the following concept (see [4])

Definition 2.2 We say that the set $K$ is second order regular at a point $y \in K$ in a direction $z \in T_{K}(y)$ with respect to a linear mapping $M: X \rightarrow Y$, if the second order tangent set $T_{K}^{2}(y, z)$ is an upper second order approximation set to $K$ at $y$ in the direction $z$ with respect to $M$. If this holds for every linear mapping $M$ and every direction $z$, we simply say that $K$ is second order regular at $y$.

Various conditions ensuring this second order regularity are discussed in [4]. In particular it is shown that the cone $\mathcal{S}_{+}^{p}$ of $p \times p$ symmetric positive semi-definite matrices is second order regular at every point $y \in \mathcal{S}_{+}^{p}$.

REMARK. Let us point out that the second order regularity used in [4] concerns the outer second order tangent sets, so that the above concept should rather be called inner second order regularity. Since in this paper we deal exclusively with the inner version, we shall omit the term "inner".

\section{Lipschitzian and Hölder directional stability of opti- mal solutions}

In this section we discuss quantitative stability of optimal or "nearly optimal" trajectories for $\left(P_{t d}\right)$. We start our discussion with Lipschitzian stability where perturbations of optimal 
solutions are of the same order as perturbations of parameters. For that purpose we need to strengthen the second order sufficient conditions (2.9) in the following way

$$
\sup _{\lambda \in S\left(D L_{d}\right)}\left\{D_{x x}^{2} L\left(x_{0}, \lambda\right)(h, h)-\sigma(\lambda, \mathcal{A}(h))\right\}>0, \forall h \in C\left(x_{0}\right) \backslash\{0\},
$$

where $\mathcal{A}(h)$ is an upper second order approximation set for $K$ at the point $G\left(x_{0}, 0\right)$ in the direction $D_{x} G\left(x_{0}, 0\right) h$ with respect to the linear mapping

$$
M(h, t):=D_{x} G\left(x_{0}, 0\right) h+t D_{u} G\left(x_{0}, 0\right) d, \quad(h, t) \in X \times \mathbb{R} .
$$

We refer to (3.1) as the strong second order sufficient conditions. These conditions were formulated for nonlinear programming problems (i.e., without the "sigma" term) in [23]. In the Banach space framework they were used in [27], also without the "sigma" term.

Let us remark that (3.1) can only hold if the set $S\left(D L_{d}\right)$ of optimal solutions of the problem $\left(D L_{d}\right)$ is non empty, which implies of course that the set $\Lambda_{0}\left(x_{0}\right)$ of Lagrange multipliers is non empty. Clearly the strong second order sufficient conditions depend on the chosen direction $d$, unless the set $\Lambda_{0}\left(x_{0}\right)$ is a singleton or the constraint mapping $G(x, u)$ does not depend on $u$. When $K$ is second order regular at $G\left(x_{0}, 0\right)$, we can take $\mathcal{A}(h)=$ $T_{K}^{2}\left(G\left(x_{0}, 0\right), D_{x} G\left(x_{0}, 0\right) h\right)$.

Theorem 3.1 Let $\bar{x}(t)$ be an $O\left(t^{2}\right)$-optimal trajectory of $\left(P_{t d}\right)$ converging, as $t \downarrow 0$, to a point $x_{0} \in \Phi\left(P_{0}\right)$ satisfying the $(D C Q)$. Suppose that

$$
v(t d) \leq v(0)+t v\left(P L_{d}\right)+O\left(t^{2}\right), \quad t \geq 0,
$$

and that the strong second order sufficient conditions (3.1) hold. Then $\bar{x}(t)$ is Lipschitz stable at $x_{0}$, i.e. for $t \geq 0$,

$$
\left\|\bar{x}(t)-x_{0}\right\|=O(t) .
$$

Proof. We argue by a contradiction. Suppose that (3.3) is false and choose a sequence $t_{k} \downarrow 0$ such that $\tau_{k} / t_{k} \rightarrow \infty$, where $\tau_{k}:=\left\|x_{k}-x_{0}\right\|$ with $x_{k}:=\bar{x}\left(t_{k}\right)$. Let $u_{k}:=t_{k} d$ and $h_{k}:=\left(x_{k}-x_{0}\right) / \tau_{k}$. Since $\left\|h_{k}\right\|=1$ and the space $X$ is finite dimensional, we can assume by passing to a subsequence if necessary, that $h_{k}$ converges to a point $h \in X \backslash\{0\}$.

Since $\bar{x}(t)$ is $O\left(t^{2}\right)$-optimal and under $(D C Q)$ we have $v(t d) \leq v(0)+O(t)$ (see [3, Part I]), we get

$$
f\left(x_{k}, u_{k}\right)-f\left(x_{0}, 0\right) \leq O\left(t_{k}\right)=o\left(\tau_{k}\right) .
$$

Since $t_{k}=o\left(\tau_{k}\right)$, the left hand side in the previous inequality is $\tau_{k} D_{x} f\left(x_{0}, 0\right) h+o\left(\tau_{k}\right)$ and hence we obtain $D_{x} f\left(x_{0}, 0\right) h \leq 0$. Moreover, since $G\left(x_{k}, u_{k}\right) \in K$ and $t_{k}=o\left(\tau_{k}\right)$, we also get $D G\left(x_{0}, 0\right) h \in T_{K}\left(G\left(x_{0}, 0\right)\right)$, and consequently $h \in C\left(x_{0}\right)$.

We can write $x_{k}$ in the form $x_{k}=x_{0}+\tau_{k} h+\frac{1}{2} \tau_{k}^{2} r_{k}$, with $\tau_{k} r_{k} \rightarrow 0$ and hence

$$
\begin{aligned}
G\left(x_{k}, u_{k}\right)= & G\left(x_{0}, 0\right)+\tau_{k} D_{x} G\left(x_{0}, 0\right) h+t_{k} D_{u} G\left(x_{0}, 0\right) d+ \\
& \frac{1}{2} \tau_{k}^{2}\left(D_{x} G\left(x_{0}, 0\right) r_{k}+D_{x x}^{2} G\left(x_{0}, 0\right)(h, h)\right)+o\left(\tau_{k}^{2}\right) .
\end{aligned}
$$

$\mathrm{RR} \quad \mathrm{n}^{\circ} 2989$ 
Since $\mathcal{A}(h)$ is a second order approximation set to $K$ at the point $G\left(x_{0}, 0\right)$ in the direction $D_{x} G\left(x_{0}, 0\right) h$ and with respect to the linear mapping $\left(D_{x} G\left(x_{0}, 0\right), D_{u} G\left(x_{0}, 0\right) d\right)$, and since $t_{k}=o\left(\tau_{k}\right)$, it follows that

$$
\begin{gathered}
2 t_{k} D_{u} G\left(x_{0}, 0\right) d+\tau_{k}^{2}\left(D_{x} G\left(x_{0}, 0\right) r_{k}+D_{x x}^{2} G\left(x_{0}, 0\right)(h, h)\right) \\
\in \tau_{k}^{2} \mathcal{A}(h)+o\left(\tau_{k}^{2}\right) B_{Y} .
\end{gathered}
$$

where $B_{Y}$ denotes the unit ball in $Y$.

Let us now use the strong second order sufficient conditions (3.1) to select $\lambda \in S\left(D L_{d}\right)$ such that

$$
D_{x x}^{2} L\left(x_{0}, \lambda\right)(h, h)-\sigma(\lambda, \mathcal{A}(h)) \geq \kappa
$$

for some $\kappa>0$. It follows from (3.5) that

$$
\begin{gathered}
\left\langle\lambda, 2 t_{k} D_{u} G\left(x_{0}, 0\right) d+\tau_{k}^{2}\left(D_{x} G\left(x_{0}, 0\right) r_{k}+D_{x x}^{2} G\left(x_{0}, 0\right)(h, h)\right)\right\rangle \\
\leq \tau_{k}^{2} \sigma(\lambda, \mathcal{A}(h))+o\left(\tau_{k}^{2}\right) .
\end{gathered}
$$

By using the second order expansion

$$
\begin{aligned}
f\left(x_{k}, u_{k}\right)= & f\left(x_{0}, 0\right)+\tau_{k} D_{x} f\left(x_{0}, 0\right) h+t_{k} D_{u} f\left(x_{0}, 0\right) d+ \\
& \frac{1}{2} \tau_{k}^{2}\left(D_{x} f\left(x_{0}, 0\right) r_{k}+D_{x x}^{2} f\left(x_{0}, 0\right)(h, h)\right)+o\left(\tau_{k}^{2}\right)
\end{aligned}
$$

together with (3.6) and (3.7), and since $D_{x} L\left(x_{0}, \lambda\right)=0$, we obtain

$$
\begin{aligned}
f\left(x_{k}, u_{k}\right)-f\left(x_{0}, 0\right) \geq & t_{k} D_{u} f\left(x_{0}, 0\right) d+\frac{\tau_{k}^{2}}{2} D_{x} f\left(x_{0}, 0\right) r_{k}+\frac{\tau_{k}^{2}}{2} D_{x x}^{2} f\left(x_{0}, 0\right)(h, h) \\
& +\frac{\tau_{k}^{2}}{2}\left\langle\lambda, \frac{2 t_{k}}{\tau_{k}^{2}} D_{u} G\left(x_{0}, 0\right) d+D_{x} G\left(x_{0}, 0\right) r_{k}+D_{x x}^{2} G\left(x_{0}, 0\right)(h, h)\right\rangle \\
& -\frac{\tau_{k}^{2}}{2} \sigma(\lambda, \mathcal{A}(h))+o\left(\tau_{k}^{2}\right), \\
= & t_{k} v\left(D L_{d}\right)+\frac{\tau_{k}^{2}}{2} D_{x x}^{2} L\left(x_{0}, \lambda\right)(h, h)-\frac{\tau_{k}^{2}}{2} \sigma(\lambda, \mathcal{A}(h))+o\left(\tau_{k}^{2}\right), \\
\geq & t_{k} v\left(D L_{d}\right)+\frac{1}{2} \kappa \tau_{k}^{2}+o\left(\tau_{k}^{2}\right) .
\end{aligned}
$$

Since $v\left(P L_{d}\right)=v\left(D L_{d}\right)$ and $t_{k}=o\left(\tau_{k}\right)$, the last inequality contradicts (3.2), and hence the proof is complete.

Remarks. Assumption (3.2) holds under the $(D C Q)$ and if the following conditions are satisfied $([3,24])$ : the linearized problem $\left(P L_{d}\right)$ has an optimal solution $\hat{h}=\hat{h}(d)$ such that for $t \geq 0$,

$$
\operatorname{dist}\left(G\left(x_{0}, 0\right)+t D G\left(x_{0}, 0\right)(\hat{h}, d), K\right)=O\left(t^{2}\right) .
$$

In case the optimal solution $\hat{h}$ exists, condition (3.9) holds if the second order tangent set $T_{K}^{2}\left(G\left(x_{0}, 0\right), D G\left(x_{0}, 0\right)(\hat{h}, d)\right)$ is non empty. If the space $X$ is reflexive (in particular finite dimensional), then existence of an optimal solution of $\left(P L_{d}\right)$ is a necessary condition for Lipschitzian stability (3.3), [3]. As we shall see in the next section, second order sufficient conditions of the type (3.1), with $\mathcal{A}(h)=T_{K}^{2}\left(G\left(x_{0}, 0\right), D_{x} G\left(x_{0}, 0\right) h\right)$, are "almost" necessary for having the Lipschitzian stability (3.3). For nonlinear programming, where the sigma term vanishes, this was already observed in [23].

INRIA 
As we already mentioned, the generalized second order sufficient conditions (2.9) do not guarantee Lipschitzian stability of optimal solutions. Nevertheless these conditions imply Hölder stability of degree $1 / 2$. The proof of the following result is similar to the previous one and is based on the upper estimate $v(t d) \leq v(0)+O(t)$ instead of the stronger bound (3.2) (cf. [3]).

Theorem 3.2 Let $\bar{x}(t)$ be an $O(t)$-optimal trajectory of $\left(P_{t d}\right)$ converging, as $t \downarrow 0$, to a point $x_{0} \in \Phi\left(P_{0}\right)$ satisfying the $(D C Q)$. Suppose that the generalized second order sufficient conditions (2.9) hold. Then for $t \geq 0$,

$$
\left\|\bar{x}(t)-x_{0}\right\|=O\left(t^{1 / 2}\right)
$$

\section{Second order expansions of the first kind}

In this section we obtain a quadratic expansion for the optimal value function $v(t d)$ and a first order expansion for optimal solutions. To obtain an upper estimate of $v(t d)$ we consider paths of the form

$$
x(t)=x_{0}+t h+\frac{1}{2} t^{2} w+o\left(t^{2}\right) .
$$

If the path $x(t)$ is feasible, an expansion of $G(x(t), t d)$ leads to (cf. [3])

$$
D G\left(x_{0}, 0\right)(h, d) \in T\left(G\left(x_{0}, 0\right)\right)
$$

and

$$
D_{x} G\left(x_{0}, 0\right) w+D^{2} G\left(x_{0}, 0\right)((h, d),(h, d)) \in \mathcal{T}_{K}^{2}(h, d),
$$

where

$$
\mathcal{T}_{K}^{2}(h, d):=T_{K}^{2}\left(G\left(x_{0}, 0\right), D G\left(x_{0}, 0\right)(h, d)\right) .
$$

A similar expansion of the objective function leads to the following optimization problem

$\left(P Q_{d, h}\right)$

$$
\begin{array}{cl}
\min _{w} & D_{x} f\left(x_{0}, 0\right) w+D^{2} f\left(x_{0}, 0\right)((h, d),(h, d)) \\
\mathrm{s.t.} & D_{x} G\left(x_{0}, 0\right) w+D^{2} G\left(x_{0}, 0\right)((h, d),(h, d)) \in \mathcal{T}_{K}^{2}(h, d) .
\end{array}
$$

By minimizing it further with respect to $h \in S\left(P L_{d}\right)$ we obtain (see [3] for details),

Proposition 4.1 Let $x_{0} \in S\left(P_{0}\right)$ be an optimal point satisfying $(D C Q)$ and suppose $\Lambda_{0}\left(x_{0}\right) \neq$ $\emptyset$. Then for $t \geq 0$,

$$
\limsup _{t \downarrow 0} \frac{v(t d)-v(0)-t v\left(P L_{d}\right)}{\frac{1}{2} t^{2}} \leq \inf _{h \in S\left(P L_{d}\right)} v\left(P Q_{d, h}\right) .
$$

The dual problem of $\left(P Q_{d, h}\right)$ can be written in the form,

$\left(D Q_{d, h}\right) \quad \max _{\lambda \in S\left(D L_{d}\right)}\left\{D^{2} L\left(x_{0}, \lambda, 0\right)((h, d),(h, d))-\sigma\left(\lambda, \mathcal{T}_{K}^{2}(h, d)\right\}\right.$. 
It is possible to show by methods of convex analysis that under $(D C Q)$ and assuming that the sets $\mathcal{T}_{K}^{2}(h, d)$ and $\Lambda_{0}\left(x_{0}\right)$ are non empty, for all $h \in S\left(P L_{d}\right)$ there is no duality gap between $\left(P Q_{d, h}\right)$ and $\left(D Q_{d, h}\right)$ and their common value is finite (cf. [21],[3]).

The upper estimate $(4.5)$ has the following lower counterpart in which the set $\mathcal{T}_{K}^{2}(h, d)$ is replaced by an upper second order approximation.

Proposition 4.2 Let $x_{0} \in S\left(P_{0}\right)$ satisfy $(D C Q)$. For each $h \in S\left(P L_{d}\right)$, let $\mathcal{A}(h, d)$ be an upper second order approximation set for $K$ at $G\left(x_{0}, 0\right)$ in the direction $D G\left(x_{0}, 0\right)(h, d)$ with respect to $D_{x} G\left(x_{0}, 0\right)$. Suppose that $\Lambda_{0}\left(x_{0}\right)$ is non empty and that there exists an $o\left(t^{2}\right)$-optimal trajectory $\bar{x}(t)$ of $\left(P_{t d}\right)$ such that $\left\|\bar{x}(t)-x_{0}\right\|=O(t)$. Then

$$
\liminf _{t \downarrow 0} \frac{v(t d)-v(0)-t v\left(P L_{d}\right)}{\frac{1}{2} t^{2}} \geq v\left(\mathcal{Q}_{d}\right),
$$

where $v\left(\mathcal{Q}_{d}\right)$ is the optimal value of the problem

$$
\min _{h \in S\left(P L_{d}\right)} \max _{\lambda \in S\left(D L_{d}\right)}\left\{D^{2} L\left(x_{0}, \lambda, 0\right)((h, d),(h, d))-\sigma(\lambda, \mathcal{A}(h, d))\right\} .
$$

Proof. The proof is similar to the one of Theorem 3.1. Consider a sequence $t_{k} \downarrow 0$ and denote $x_{k}:=\bar{x}\left(t_{k}\right), h_{k}:=t_{k}^{-1}\left(x_{k}-x_{0}\right)$ and $u_{k}:=t_{k} d$. Since $\bar{x}(t)$ is Lipschitz stable at $x_{0}$ we have that $h_{k}$ are bounded so that, passing to a subsequence if necessary, we may assume that $h_{k} \rightarrow h$ for some $h \in X$, for which it is not difficult to show that $h \in S\left(P L_{d}\right)$ (see [3]).

We can write $x_{k}=x_{0}+t_{k} h+\frac{1}{2} t_{k}^{2} r_{k}$ with $t_{k} r_{k} \rightarrow 0$, and using the second order expansion

$$
\begin{aligned}
G\left(x_{k}, u_{k}\right)= & G\left(x_{0}, 0\right)+t_{k} D G\left(x_{0}, 0\right)(h, d)+ \\
& \frac{1}{2} t_{k}^{2}\left(D_{x} G\left(x_{0}, 0\right) r_{k}+D^{2} G\left(x_{0}, 0\right)((h, d),(h, d))+o\left(t_{k}^{2}\right),\right.
\end{aligned}
$$

we may deduce

$$
D_{x} G\left(x_{0}, 0\right) r_{k}+D^{2} G\left(x_{0}, 0\right)((h, d),(h, d))+o(1) \in \mathcal{A}(h, d) .
$$

Similarly we also get

$$
\begin{aligned}
& v\left(u_{k}\right)-v(0)=f\left(x_{k}, u_{k}\right)-f\left(x_{0}, 0\right)+o\left(t_{k}^{2}\right)= \\
& t_{k} v\left(P L_{d}\right)+\frac{1}{2} t_{k}^{2}\left[D_{x} f\left(x_{0}, 0\right) r_{k}+D^{2} f\left(x_{0}, 0\right)((h, d),(h, d))\right]+o\left(t_{k}^{2}\right) .
\end{aligned}
$$

Let $\lambda \in S\left(D L_{d}\right)$. It follows from (4.8) that

$$
\left\langle\lambda, D_{x} G\left(x_{0}, 0\right) r_{k}+D^{2} G\left(x_{0}, 0\right)((h, d),(h, d))\right\rangle-\sigma(\lambda, \mathcal{A}(h, d)) \leq o(1),
$$

where the term $o(1)$ can be taken independently of $\lambda$ since $S\left(D L_{d}\right)$ is bounded. By adding half times $t_{k}^{2}$ of the above term to the right hand side of (4.9), and since $D_{x} L\left(x_{0}, \lambda, 0\right)=0$, we obtain

$$
\begin{aligned}
v\left(u_{k}\right) \geq & v(0)+t_{k} v\left(P L_{d}\right)+ \\
& \frac{1}{2} t_{k}^{2}\left[D^{2} L\left(x_{0}, \lambda, 0\right)((h, d),(h, d))-\sigma(\lambda, \mathcal{A}(h, d))\right]+o\left(t_{k}^{2}\right) .
\end{aligned}
$$


Since $\lambda \in S\left(D L_{d}\right)$ was arbitrary and $h \in S\left(P L_{d}\right)$, we obtain (4.6) as claimed.

Recall that sufficient conditions for Lipschitzian stability of optimal solutions are discussed in Theorem 3.1. The lower estimate (4.6) coincides with the upper estimate (4.5) if for every $h \in S\left(P L_{d}\right)$ the second order tangent set $\mathcal{T}_{K}^{2}(h, d)$ can serve as an upper second order approximation set, that is, under the additional condition of second order regularity. Thus we deduce the following second order expansion of the optimal value function.

Theorem 4.1 Let $x_{0} \in S\left(P_{0}\right)$ satisfy $(D C Q)$. Suppose that $\Lambda_{0}\left(x_{0}\right)$ is non empty, that there exists an o( $\left.t^{2}\right)$-optimal trajectory $\bar{x}(t)$ of $\left(P_{t d}\right)$ such that $\left\|\bar{x}(t)-x_{0}\right\|=O(t)$, and that for every $h \in S\left(P L_{d}\right)$ the set $K$ is second order regular at $G\left(x_{0}, 0\right)$ in the direction $D G\left(x_{0}, 0\right)(h, d)$ with respect to $D_{x} G\left(x_{0}, 0\right)$. Then:

(i) For $t \geq 0$,

$$
v(t d)=v(0)+t v\left(P L_{d}\right)+\frac{1}{2} t^{2} v\left(Q_{d}\right)+o\left(t^{2}\right)
$$

where $v\left(Q_{d}\right) \in \mathbb{R}$ is the optimal value of the problem

$$
\min _{h \in S\left(P L_{d}\right)} \max _{\lambda \in S\left(D L_{d}\right)}\left\{D^{2} L\left(x_{0}, \lambda, 0\right)((h, d),(h, d))-\sigma\left(\lambda, \mathcal{T}_{K}^{2}(h, d)\right)\right\} .
$$

(ii) Every accumulation point $\bar{h}$ of $\left(x(t)-x_{0}\right) / t$, with $t \downarrow 0$ and $x(t)$ being an o( $\left.t^{2}\right)$-optimal trajectory of $\left(P_{t d}\right)$, is an optimal solution of the problem $\left(Q_{d}\right)$. If in addition $\lambda(t)$ is a Lagrange multiplier associated with $x(t)$, then every weak* accumulation point of $\lambda(t)$ belongs to $S\left(D L_{d}\right)$.

(iii) Let $\bar{h}$ be an optimal solution of $\left(Q_{d}\right)$ and let $\bar{w}$ be a corresponding optimal solution of $\left(P Q_{d, h}\right)$ (assuming that such optimal solutions exist). Then there exists an o( $\left.t^{2}\right)$-optimal trajectory for $\left(P_{t d}\right)$ of the form $\hat{x}(t):=x_{0}+t \bar{h}+\frac{1}{2} t^{2} \bar{w}+o\left(t^{2}\right)$.

Proof. From propositions 4.1 and 4.2 it follows that

$$
\liminf _{t \downarrow 0} \frac{v(t d)-v(0)-t v\left(P L_{d}\right)}{\frac{1}{2} t^{2}}=v\left(Q_{d}\right) .
$$

From the computation in the proof of proposition 4.2, it follows that any limit point of $t^{-1}\left(\bar{x}(t)-x_{0}\right)$ is solution of $\left(Q_{d}\right)$. As $\sigma\left(\lambda, \mathcal{T}_{K}^{2}(h, d)\right) \leq 0$ ([3, Part II, Lemma 2.1]), we have $v\left(Q_{d}\right) \in \mathbb{R}$. This proves $(i)$.

If $\bar{h}$ is an accumulation point of $\left(x(t)-x_{0}\right) / t$, then as we mentioned in the proof of proposition $4.2, \bar{h} \in S\left(P L_{d}\right)$ and the first part of statement (ii) follows from (4.11) and (4.5). Let $\bar{\lambda}$ be a weak* accumulation point of $\lambda(t)$, where $\lambda(t)$ is a Lagrange multiplier associated with $x(t)$. It is easily proved that $\bar{\lambda}$ is a Lagrange multiplier associated with $x_{0}$. From the inequalities $\left\langle\bar{\lambda}, G(x(t), t d)-G\left(x_{0}, 0\right)\right\rangle \leq 0$ and $\left\langle\lambda(t), G(x(t), t d)-G\left(x_{0}, 0\right)\right\rangle \geq 0$, and a first order expansion of $G(x(t), t d)-G\left(x_{0}, 0\right)$, we obtain $\left\langle\bar{\lambda}, G^{\prime}\left(x_{0}, 0\right)(\bar{h}, d)\right\rangle=0$. As $\bar{h}$ is feasible for $\left(P L_{d}\right)$, and $\bar{\lambda}$ is feasible for the dual problem $\left(D L_{d}\right)$, statement (ii) follows. Because of $(D C Q)$, it follows by [3, Part I, Theorem B.2] that there exists a feasible path $\hat{x}(t)$ of $\left(P_{t d}\right)$ of the form specified in (iv). Moreover, $f(\hat{x}(t), t d)=v(t d)+o\left(t^{2}\right)$ and hence statement (iv) follows.

The following is an immediate consequence of the assertion (ii) in the above theorem.

RR $\quad n^{\circ} 2989$ 
Corollary 4.1 Suppose that, in addition to the assumptions of theorem 4.1, problem $\left(Q_{d}\right)$ possesses a unique optimal solution $h^{*}$. Let $\bar{x}(t)$ be an o( $\left.t^{2}\right)$-optimal trajectory of $\left(P_{t d}\right)$ such that $\left\|\bar{x}(t)-x_{0}\right\|=O(t)$. Then $\bar{x}(t)$ is right side differentiable at $t=0$ and the corresponding right side derivative is equal to $h^{*}$.

Let us give a sufficient condition for uniqueness of the optimal solution of the problem $\left(Q_{d}\right)$. Let us observe first that for any $\lambda$, the function $\psi(z):=-\sigma\left(\lambda, T_{K}^{2}(y, z)\right)$ is convex. Indeed, consider $z_{1}, z_{2} \in Y$ and let $w_{1} \in T_{K}^{2}\left(y, z_{1}\right), w_{2} \in T_{K}^{2}\left(y, z_{2}\right)$ and $\alpha \in[0,1]$. Then, by convexity of $K$, we have that

$$
\alpha w_{1}+(1-\alpha) w_{2} \in T_{K}^{2}\left(y, \alpha z_{1}+(1-\alpha) z_{2}\right)
$$

and hence

$$
\alpha\left\langle\lambda, w_{1}\right\rangle+(1-\alpha)\left\langle\lambda, w_{2}\right\rangle \leq \sigma\left(\lambda, T_{K}^{2}\left(y, \alpha z_{1}+(1-\alpha) z_{2}\right)\right) .
$$

Since $w_{1}$ and $w_{2}$ were arbitrary elements of the corresponding second order tangent sets, we obtain that the function $\sigma\left(\lambda, T_{K}^{2}(y, \cdot)\right)$ is concave, and hence $\psi(\cdot)$ is convex. It follows that if, for every $\lambda \in S\left(D L_{d}\right)$, the Hessian $D_{x x}^{2} L\left(x_{0}, \lambda, 0\right)$ is positive definite over the linear space generated by the (convex) set $S\left(P L_{d}\right)$, then the max-function of the problem $\left(Q_{d}\right)$ is strictly convex over this linear space and hence $S\left(Q_{d}\right)$ is a singleton.

The right side derivative of $\bar{x}(t)$, at $t=0$, can be viewed as the directional derivative, in the direction $d$, of the corresponding $o\left(\|u\|^{2}\right)$-optimal solution $\bar{x}(u)$ of $\left(P_{u}\right)$. Note also that if there exists an $O\left(t^{2}\right)$-optimal trajectory $\bar{x}(t)$ of $\left(P_{t d}\right)$ which is Lipschitz stable at $x_{0}$, then for $\lambda \in S\left(D L_{d}\right)$,

$$
\begin{aligned}
v(t d) & =f(\bar{x}(t), t d)+O\left(t^{2}\right) \\
& \geq f(\bar{x}(t), t d)+\left\langle\lambda, G(\bar{x}(t), t d)-G\left(x_{0}, 0\right)\right\rangle+O\left(t^{2}\right) \\
& =f\left(x_{0}, 0\right)+L(\bar{x}(t), \lambda, t d)-L\left(x_{0}, \lambda, 0\right)+O\left(t^{2}\right) \\
& \geq v(0)+t v\left(P L_{d}\right)+O\left(t^{2}\right) .
\end{aligned}
$$

The above inequality and (4.5) can hold together only if $\inf _{h \in S\left(P L_{d}\right)} v\left(P Q_{d, h}\right)>-\infty$. Since $C\left(x_{0}\right)$ is the recession cone of $S\left(P L_{d}\right)$ (provided $S\left(P L_{d}\right)$ is non empty), and since under $(D C Q)$ we have $v\left(P Q_{d, h}\right)=v\left(D Q_{d, h}\right)$, we obtain that under these assumptions the following second order conditions

$$
\sup _{\lambda \in S\left(D L_{d}\right)}\left\{D_{x x}^{2} L\left(x_{0}, \lambda\right)(h, h)-\sigma\left(\lambda, \mathcal{T}_{K}^{2}(h)\right)\right\} \geq 0, \forall h \in C\left(x_{0}\right),
$$

where $\mathcal{T}_{K}^{2}(h)=T_{K}^{2}\left(G\left(x_{0}, 0\right), D_{x} G\left(x_{0}, 0\right) h\right)$, are necessary for Lipschitzian stability of optimal solutions. Note the similarity of (4.15) with the strong second order conditions (3.1).

Theorem 4.1 extends previous results obtained in the framework of nonlinear programming $[1,5,23]$. The main difference between the results presented in Theorem 4.1 and those obtained in the nonlinear programming setting is the additional curvature term ("sigma" term) and the assumption of nonemptiness of $S\left(P L_{d}\right)$. The condition $(D C Q)$ and the existence of Lagrange multipliers (which follows from the strong second order sufficient conditions) imply that $\left(P L_{d}\right)$ has a finite value and that its dual $\left(D L_{d}\right)$ has an optimal solution.

INRIA 
In the nonlinear programming setting, the corresponding optimization problem $\left(P L_{d}\right)$ is linear and it has optimal solutions as soon as its optimal value is finite. However, for general non polyhedral sets $K$ we have to postulate that $S\left(P L_{d}\right)$ is non empty. If this is not true, then as we mentined earlier, $o(t)$-optimal trajectories are not Lipschitz stable. In that case we may apply the analysis of the second kind that follows.

\section{Expansion of the second kind}

In this section we discuss situations where approximate optimal solutions are Hölder stable of degree $1 / 2$ (see Theorem 3.2) and the set of Lagrange multipliers is non empty. Then it is convenient to consider paths of the form

$$
x(t):=x_{0}+t^{1 / 2} h+t z+o(t) .
$$

It follows that

$$
\begin{aligned}
f(x(t), t d)= & f\left(x_{0}, 0\right)+t^{1 / 2} D_{x} f\left(x_{0}, 0\right) h+ \\
& \frac{1}{2} t\left[2 D f\left(x_{0}, 0\right)(z, d)+D_{x x}^{2} f\left(x_{0}, 0\right)(h, h)\right]+o(t) .
\end{aligned}
$$

Expanding $G(x(t), t d)$ in a similar way we obtain that if the path $x(t)$ is feasible, then

$$
D_{x} G\left(x_{0}, 0\right) h \in T\left(G\left(x_{0}, 0\right)\right)
$$

and

$$
2 D G\left(x_{0}, 0\right)(z, d)+D_{x x}^{2} G\left(x_{0}, 0\right)(h, h) \in \mathcal{T}_{K}^{2}(h) .
$$

where

$$
\mathcal{T}_{K}^{2}(h):=T_{K}^{2}\left(G\left(x_{0}, 0\right), D_{x} G\left(x_{0}, 0\right) h\right) .
$$

We need an additional assumption. We say that the strong directional constraint qualification $(S D C Q)$ holds if $(D C Q)$ is satisfied and, given a path $x(t)$ of the form (5.1) and such that (5.3) and (5.4) hold, then for $\gamma<1$ close to 1 one can find $z_{\gamma} \in X$ with $z_{\gamma} \rightarrow z$, and a feasible path $x_{\gamma}(t)$ satisfying

$$
x_{\gamma}(t)=x_{0}+\gamma t^{1 / 2} h+t z_{\gamma}+o(t) .
$$

In [3, Part II], where this assumption was introduced, it was proved that $(S D C Q)$ is a consequence of $(D C Q)$ whenever the set $K$ has a nonempty interior, which is the case for semi-definite and semi-infinite optimization. Note also that $(S D C Q)$ is satisfied whenever $(C Q)$ holds.

Consider the problem

$$
\operatorname{Min}_{h \in C\left(x_{0}\right)}\left\{\begin{array}{cl}
\operatorname{Min}_{z \in X} & 2 D f\left(x_{0}, 0\right)(z, d)+D_{x x}^{2} f\left(x_{0}, 0\right)(h, h) \\
\text { s.t. } & 2 D G\left(x_{0}, 0\right)(z, d)+D_{x x}^{2} G\left(x_{0}, 0\right)(h, h) \in \mathcal{T}_{K}^{2}(h)
\end{array}\right\},
$$

and its dual

$$
\underset{h \in C\left(x_{0}\right)}{\operatorname{Min}} \operatorname{Max}_{\lambda \in \Lambda_{0}\left(x_{0}\right)}\left\{2 D_{u} L\left(x_{0}, \lambda, 0\right) d+D_{x x}^{2} L\left(x_{0}, \lambda, 0\right)(h, h)-\sigma\left(\lambda, \mathcal{T}_{K}^{2}(h)\right)\right\}
$$

$\mathrm{RR} \quad \mathrm{n}^{\circ} 2989$ 
Theorem 5.1 Let $\bar{x}(t)$ be an o(t)-optimal trajectory for $\left(P_{t d}\right)$ converging to a point $x_{0} \in$ $S\left(P_{0}\right)$ satisfying $(S D C Q)$. Suppose that the set of Lagrange multipliers $\Lambda_{0}\left(x_{0}\right)$ is non empty, that for every $h \in C\left(x_{0}\right)$ the set $K$ is second order regular at $G\left(x_{0}, 0\right)$ in the direction $D_{x} G\left(x_{0}, 0\right) h$ with respect to $D_{x} G\left(x_{0}, 0\right)$, and that the following (weak) second order conditions hold

$$
\sup _{\lambda \in \Lambda_{0}\left(x_{0}\right)}\left\{D_{x x}^{2} L\left(x_{0}, \lambda, 0\right)(h, h)-\sigma\left(\lambda, \mathcal{T}_{K}^{2}(h)\right)\right\}>0, \quad \forall h \in C\left(x_{0}\right) \backslash\{0\} .
$$

Then:

(i) $\bar{x}(t)$ is Hölder stable of degree 1/2, i.e. $\left\|\bar{x}(t)-x_{0}\right\|=O\left(t^{1 / 2}\right)$.

(ii) There is no duality gap between problems $\left(\mathcal{Q}_{d}^{2}\right)$ and $\left(\mathcal{D}_{d}^{2}\right)$, and their common value $v\left(\mathcal{Q}_{d}^{2}\right)=v\left(\mathcal{D}_{d}^{2}\right)$ is less than or equal to $2 v\left(P L_{d}\right)$.

(iii) The optimal value function can be expanded as

$$
v(t d)=v(0)+\frac{1}{2} t v\left(\mathcal{D}_{d}^{2}\right)+o(t) .
$$

(iv) If $h \in X$ is an accumulation point of $t^{-1 / 2}\left(\bar{x}(t)-x_{0}\right)$, then $h \in S\left(\mathcal{D}_{d}^{2}\right)$.

(v) If $\left(\mathcal{Q}_{d}^{2}\right)$ has an optimal solution $(\bar{h}, \bar{z})$ and $(C Q)$ holds, then there exists an o(t)-optimal trajectory $x(t)$ of $\left(P_{t d}\right)$ such that $x(t)=x_{0}+t^{1 / 2} \bar{h}+o\left(t^{1 / 2}\right)$.

Proof. By the second order conditions (5.7) and the second order regularity of the set $K$, statement (i) follows from Theorem 3.2. Assertion (ii) and the upper estimate

$$
v(t d) \leq v(0)+\frac{1}{2} t v\left(\mathcal{D}_{d}^{2}\right)+o(t) .
$$

are consequences of [3, Part II, Theorem 3.1]. To prove the converse inequality in (5.9) let $t_{k} \downarrow 0$ be an arbitrary sequence. Letting $x_{k}:=\bar{x}\left(t_{k}\right)$ and using part (i), by passing to a subsequence if necessary, we may assume that $t_{k}^{-1 / 2}\left(x_{k}-x_{0}\right)$ converges towards some $h \in X$. Hence we may write $x_{k}=x_{0}+t_{k}^{1 / 2} h+t_{k} z_{k}$, with $t_{k}^{1 / 2} z_{k} \rightarrow 0$. An expansion of $G\left(x_{k}, t_{k} d\right)$ similar to (5.2) and the second order regularity of $K$ lead to $D_{x} G\left(x_{0}, 0\right) h \in T_{K}\left(G\left(x_{0}, 0\right)\right)$ and

$$
2 D G\left(x_{0}, 0\right)\left(z_{k}, d\right)+D_{x x}^{2} G\left(x_{0}, 0\right)(h, h)+o(1) \in \mathcal{T}_{K}^{2}(h) .
$$

Since

$$
f\left(x_{k}, t_{k} d\right)=v\left(t_{k} d\right)+o\left(t_{k}\right) \leq v(0)+t_{k} v\left(P L_{d}\right)+o\left(t_{k}\right)=f\left(x_{0}, 0\right)+o\left(t_{k}^{1 / 2}\right),
$$

we have $D_{x} f\left(x_{0}, 0\right) h \leq 0$, and therefore $h \in C\left(x_{0}\right)$.

Let $\lambda \in \Lambda_{0}\left(x_{0}\right)$. Using $D_{x} L\left(x_{0}, \lambda, 0\right)=0,\left\langle\lambda, D_{x} G\left(x_{0}, 0\right) h\right\rangle=0$ and (5.10), we obtain

$$
\begin{aligned}
v\left(t_{k} d\right)= & f\left(x_{k}, t_{k} d\right)+o\left(t_{k}\right), \\
= & f\left(x_{0}, 0\right)+L\left(x_{k}, \lambda, t_{k} d\right)-L\left(x_{0}, \lambda, 0\right)-\left\langle\lambda, G\left(x_{k}, t_{k} d\right)-G\left(x_{0}, 0\right)\right\rangle+o\left(t_{k}\right), \\
= & v(0)+\frac{1}{2} t_{k}\left[2 D_{u} L\left(x_{0}, \lambda, 0\right) d+D_{x x}^{2} L\left(x_{0}, \lambda, 0\right)(h, h)\right] \\
& -\frac{1}{2} t_{k}\left\langle\lambda, 2 D G\left(x_{0}, 0\right)\left(z_{k}, d\right)+D_{x x}^{2} G\left(x_{0}, 0\right)(h, h)\right\rangle+o\left(t_{k}\right), \\
\geq & v(0)+\frac{1}{2} t_{k}\left[2 D_{u} L\left(x_{0}, \lambda, 0\right) d+D_{x x}^{2} L\left(x_{0}, \lambda, 0\right)(h, h)-\sigma\left(\lambda, \mathcal{T}_{K}^{2}(h)\right]+o\left(t_{k}\right) .\right.
\end{aligned}
$$

INRIA 
This being true for any $\lambda \in \Lambda_{0}\left(x_{0}\right)$, we obtain

$$
v\left(t_{k} d\right) \geq v(0)+\frac{t_{k}}{2} \sup _{\lambda \in \Lambda_{0}\left(x_{0}\right)}\left\{2 D_{u} L\left(x_{0}, \lambda, 0\right) d+D_{x x}^{2} L\left(x_{0}, \lambda, 0\right)(h, h)-\sigma\left(\lambda, \mathcal{T}_{K}^{2}(h)\right)\right\}+o\left(t_{k}\right) .
$$

Together with (5.9) this proves (5.8), i.e. (iii), as well as $h \in S\left(\mathcal{D}_{d}^{2}\right)$, i.e. (iv).

It remains to prove $(v)$. It follows from $(C Q)$ that there exists a feasible path of the form $x(t)=x_{0}+t^{1 / 2} \bar{h}+t \bar{z}+o(t)$. Computing the expansion of $f(x(t), t d)$, we find that $x(t)$ is an $o(t)$-optimal trajectory, and the proof is complete.

It may be surprising to observe that under quite natural assumptions (directional constraint qualification and the standard second order sufficient conditions), the first order expansion (5.8) of the optimal value function involves the second order information, included in the problem $\left(\mathcal{D}_{d}^{2}\right)$, and perturbed optimal solutions are not Lipschitz stable. This is already true for nonlinear programming problems. In that case the above theorem reduces to results presented in [12] and [5]. The upper estimate (5.9) was obtained in the Banach space framework in [3].

Note that if the second order conditions (4.15) hold, then $h=0$ and any $\lambda \in S\left(D L_{d}\right)$ are optimal solutions of the problem $\left(\mathcal{D}_{d}^{2}\right)$ and hence $v\left(\mathcal{D}_{d}^{2}\right)=2 v\left(D L_{d}\right)$. Therefore we obtain the following

Corollary 5.1 Suppose that the assumptions of Theorem 5.1 hold as well as the second order conditions (4.15). Then for $t \geq 0$,

$$
v(t d)=v(0)+t v\left(D L_{d}\right)+o(t) .
$$

\section{Expansion of the third kind}

In this section we discuss a situation similar to the one considered in the previous section except that the set of Lagrange multipliers is assumed to be empty. We consider a point $x_{0}$ satisfying the Fritz John optimality conditions, i.e. we assume that the set $\Lambda_{0}^{g}\left(x_{0}\right)$ of generalized Lagrange multipliers is non empty. These two conditions, $\Lambda_{0}\left(x_{0}\right)=\emptyset$ and $\Lambda_{0}^{g}\left(x_{0}\right) \neq \emptyset$, imply that if $(\alpha, \lambda) \in \Lambda_{0}^{g}\left(x_{0}\right)$, then $\alpha=0$. We consider again paths of the form (5.1). The main difference from the previous case is that now variations of the optimal value function are of order $O\left(t^{1 / 2}\right)$, and for $h \in C\left(x_{0}\right), D_{x} f\left(x_{0}, 0\right) h$ can be negative. Consider the following subset of the critical cone

$$
C^{2}\left(x_{0}\right)=\left\{h \in C\left(x_{0}\right): \exists z \in X \text { s.t. } 2 D G\left(x_{0}, 0\right)(z, d)+D_{x x}^{2} G\left(x_{0}, 0\right)(h, h) \in \mathcal{T}_{K}^{2}(h)\right\} .
$$

It is worth pointing out that when $\Lambda_{0}\left(x_{0}\right)=\emptyset$ and $\Lambda_{0}^{g}\left(x_{0}\right) \neq \emptyset$, and assuming $(S D C Q)$, a point $h$ belongs to $C^{2}\left(x_{0}\right)$ if and only if (see [3, Part II])

$$
2 D_{u} L^{g}\left(x_{0}, 0, \lambda, 0\right) d+D_{x x}^{2} L^{g}\left(x_{0}, 0, \lambda, 0\right)(h, h) \leq \sigma\left(\lambda, T_{K}^{2}(h)\right), \quad \forall(0, \lambda) \in \Lambda_{0}^{g}\left(x_{0}\right) .
$$

$\mathrm{RR} \mathrm{n}^{\circ} 2989$ 
Theorem 6.1 Let $\bar{x}(t)$ be an o(t)-optimal trajectory of $\left(P_{t d}\right)$ converging to a point $x_{0} \in$ $S\left(P_{0}\right)$ satisfying the Fritz John optimality conditions (2.3) and the (SDCQ). Suppose that the set of Lagrange multipliers $\Lambda_{0}\left(x_{0}\right)$ is empty, that for every $h \in C\left(x_{0}\right)$ the set $K$ is second order regular at $G\left(x_{0}, 0\right)$ in the direction $D_{x} G\left(x_{0}, 0\right) h$ with respect to $D_{x} G\left(x_{0}, 0\right)$, and that the generalized second order sufficient conditions (2.9) hold with $\mathcal{A}(h)=\mathcal{T}_{K}^{2}(h)$. Then:

(i) $\bar{x}(t)$ is Hölder stable of degree 1/2, i.e. $\left\|\bar{x}(t)-x_{0}\right\|=O\left(t^{1 / 2}\right)$.

(ii) The optimal value function may be expanded as

$$
v(t d)=v(0)+t^{1 / 2} v\left(\mathcal{Q}_{d}^{3}\right)+o\left(t^{1 / 2}\right),
$$

where $v\left(\mathcal{Q}_{d}^{3}\right)$ is the optimal value of the problem

$$
\operatorname{Min}_{h} D_{x} f\left(x_{0}, 0\right) h \quad \text { s.t. } h \in C^{2}\left(x_{0}\right) .
$$

(iii) If $h$ is an accumulation point of $t^{-1 / 2}\left(\bar{x}(t)-x_{0}\right)$, then $h \in S\left(\mathcal{Q}_{d}^{3}\right)$.

The proof of this theorem is similar to that of Theorem 5.1 and will be omitted (see also [3, Part II]). Theorem 6.1 extends similar results obtained for nonlinear programming problems in [2].

\section{Applications and examples}

In this section we discuss some applications and particular examples of the developed theory.

\subsection{Second order regularity in semi-infinite programming}

Let us first show that Theorem 4.1 itself can be used to verify second order regularity of a set defined by an infinite number of inequalities. Suppose that for every $x \in X$ the function $f(x, \cdot): U \rightarrow \mathbb{R}$ is concave and that the mapping $G(x)$ does not depend on $u$, and consider the set

$$
\Theta:=\{u \in U: f(x, u) \geq 0, \forall x \in \Phi\},
$$

where $\Phi:=\{x \in X: G(x) \in K\}$. Since the functions $f(x, \cdot)$ are concave, the set $\Theta$ is convex. Clearly the set $\Theta$ can be also defined in the form $\Theta=\{u: v(u) \geq 0\}$, where $v(u):=\inf _{x \in \Phi} f(x, u)$ is the corresponding optimal value function.

Consider a point $u_{0} \in \Theta$ and denote $\Delta_{0}:=\left\{x \in \Phi: f\left(x, u_{0}\right)=0\right\}$ the set of minimizers of $f\left(\cdot, u_{0}\right)$ over $\Phi$. Note that $\Delta_{0} \neq \emptyset$ iff $v\left(u_{0}\right)=0$ and in the later case the set $\Delta_{0}$ is the set of minimizers of $f\left(\cdot, u_{0}\right)$ over $\Phi$. Suppose that there exists a compact set $\Sigma$ such that for all $u$ in a neighborhood of $u_{0}$ and some $\alpha>v\left(u_{0}\right)$,

$$
\{x \in \Phi: f(x, u) \leq \alpha\} \subset \Sigma .
$$


We refer to this assumption as the inf-compactness condition. By Danskin theorem [11], under the inf-compactness condition, the optimal value function $v(u)$ is directionally differentiable at $u_{0}$ and, provided $v\left(u_{0}\right)=0$, its directional derivative is given by

$$
v^{\prime}\left(u_{0}, d\right)=\inf _{x \in \Delta_{0}} D_{u} f\left(x, u_{0}\right) d .
$$

We assume that $\Delta_{0}$ is non empty and denote

$$
\Delta_{1}(d):=\left\{x \in \Delta_{0}: D_{u} f\left(x, u_{0}\right) d=v^{\prime}\left(u_{0}, d\right)\right\} .
$$

We also assume that the following second order growth condition holds for the function $f\left(\cdot, u_{0}\right)$ : there exist a neighborhood $N$ of $\Delta_{0}$ and $c>0$ such that

$$
f\left(x, u_{0}\right) \geq c \operatorname{dist}\left(x, \Delta_{0}\right)^{2}, \quad \forall x \in \Phi \cap N .
$$

Theorem 7.1 Suppose that the Slater condition holds, i.e. there is $\bar{u}$ such that $f(x, \bar{u})>0$ for all $x \in \Phi$, that the set $\Delta_{0}$ is non empty and finite, that the inf-compactness condition and the second order growth condition (7.3) hold, that for every $x_{0} \in \Delta_{0}$ the (CQ) holds and for every $h \in C\left(x_{0}\right)$ the set $K$ is second order regular at $G\left(x_{0}\right)$ in the direction $D G\left(x_{0}\right) h$ with respect to $D G\left(x_{0}\right)$. Then the set $\Theta$ is second order regular at $u_{0}$ and, if in addition $v^{\prime}\left(u_{0}, d\right)=0$, then

$$
T_{\Theta}^{2}\left(u_{0}, d\right)=\left\{w \in U: D_{u} f\left(x_{0}, u_{0}\right) w+\kappa\left(x_{0}, d\right) \geq 0, \forall x_{0} \in \Delta_{1}(d)\right\},
$$

where $\kappa\left(x_{0}, d\right)$ is the optimal value of the problem

$$
\operatorname{Min}_{h \in C\left(x_{0}\right)} \operatorname{Max}_{\lambda \in \Lambda_{0}\left(x_{0}\right)}\left\{D^{2} L\left(x_{0}, \lambda, u_{0}\right)((h, d),(h, d))-\sigma\left(\lambda, \mathcal{T}_{K}^{2}(h)\right)\right\} .
$$

Proof. Consider a point $x_{0} \in \Delta_{0}$. As we mentioned earlier, $x_{0}$ is a minimizer of $f\left(\cdot, u_{0}\right)$ subject to the constraint $G(\cdot) \in K$ and hence can be viewed as an optimal solution of the corresponding (unperturbed) problem $\left(P_{u_{0}}\right)$. Note that in the present case the constraint mapping $G(\cdot)$ does not depend on $u$. By restricting the optimization problem to a neighborhhod of $x_{0}$, we obtain from Theorem 4.1 that the corresponding optimal value function $\hat{v}(u)$ can be expanded as

$$
\hat{v}\left(u_{0}+t d+\frac{1}{2} t^{2} w\right)=f\left(x_{0}, u_{0}\right)+t D_{u} f\left(x_{0}, u_{0}\right) d+\frac{1}{2} t^{2} \xi_{x_{0}}(d, w)+o\left(t^{2}\right),
$$

where $\xi_{x_{0}}(d, w)$ is the optimal solution of the problem

$$
\operatorname{Min}_{h \in C\left(x_{0}\right)} \operatorname{Max}_{\lambda \in \Lambda_{0}\left(x_{0}\right)}\left\{D_{u} L\left(x_{0}, \lambda, u_{0}\right) w+D^{2} L\left(x_{0}, \lambda, u_{0}\right)((h, d),(h, d))-\sigma\left(\lambda, \mathcal{T}_{K}^{2}(h)\right)\right\} .
$$

The additional term $D_{u} L\left(x_{0}, \lambda, u_{0}\right) w$ in the above expansion appears since the optimal value function is expanded now along the parabolic curve (with the additional term $\frac{1}{2} t^{2} w$ ), and this term is equal to $D_{u} f\left(x_{0}, u_{0}\right) w$ since $G(\cdot)$ does not depend on $u$. Note also that the set 
$\Lambda_{0}\left(x_{0}\right)$ of the corresponding Lagrange multipliers is non empty because of the $(C Q)$ and that existence of Lipschitz stable optimal solutions is ensured by the second order growth and inf-compactness conditions. Such analysis can be performed at every point of the set $\Delta_{0}$ and the optimal value function $v(u)$ is given by the minimum of the optimal value functions corresponding to points $x_{0} \in \Delta_{0}$. Consequently we obtain that the optimal value function $v(u)$ is second order directionally differentiable and its second order directional derivative $v^{\prime \prime}\left(u_{0}, d, w\right)$ is given by the minimum of functions $\xi_{x_{0}}(d, w), x_{0} \in \Delta_{1}(d)$. Because of the Slater condition this implies (7.4), as proved in [4].

Note that, under the Slater condition, $T_{\Theta}\left(u_{0}\right)=\left\{d \in U: v^{\prime}\left(u_{0}, d\right) \geq 0\right\}$. Therefore if $v^{\prime}\left(u_{0}, d\right)<0$, then $T_{\Theta}^{2}\left(u_{0}, d\right)$ is empty. Also if $v^{\prime}\left(u_{0}, d\right)>0$, then $T_{\Theta}^{2}\left(u_{0}, d\right)$ is the whole space $U$. Consequently, in order to show second order regularity of $\Theta$, we have to consider only the case when $v^{\prime}\left(u_{0}, d\right)=0$. Consider a sequence $u_{k}=u_{0}+t_{k} d+\frac{1}{2} t_{k}^{2} r_{k}$ such that $t_{k} \downarrow 0, t_{k} r_{k} \rightarrow 0$ and $v\left(u_{k}\right) \geq 0$, i.e. $u_{k} \in \Theta$. Consider also a point $x_{0} \in \Delta_{1}(d)$ and a path

$x(t)=x_{0}+t h+\frac{1}{2} t^{2} z+o\left(t^{2}\right)$ for some $h \in C\left(x_{0}\right)$. By using the second order expansion of $G(x(t))$, it is not difficult to see that such a path can be feasible, for small $t \geq 0$, only if

$$
D G\left(x_{0}\right) z+D^{2} G\left(x_{0}\right)(h, h) \in \mathcal{T}_{K}^{2}(h) .
$$

By the Robinson-Ursescu stability theorem, because of the $(C Q)$, the above condition is also sufficient for existence of such feasible path. Let $x_{k}:=x\left(t_{k}\right)=x_{0}+t_{k} h+\frac{1}{2} t_{k}^{2} z_{k}$ be such that $G\left(x_{k}\right) \in K$ and $z_{k} \rightarrow z$. Then, since $f\left(x_{0}, u_{0}\right)=v\left(u_{0}\right)=0, h \in C\left(x_{0}\right)$ and hence $D_{x} f\left(x_{0}, u_{0}\right) h=0, D_{u} f\left(x_{0}, u_{0}\right) d=v^{\prime}\left(u_{0}, d\right)=0$, we obtain

$$
f\left(x_{k}, u_{k}\right)=\frac{1}{2} t_{k}^{2}\left[D f\left(x_{0}, u_{0}\right)\left(z, r_{k}\right)+D^{2} f\left(x_{0}, u_{0}\right)((h, d),(h, d))\right]+o\left(t_{k}^{2}\right),
$$

where the term $o\left(t_{k}^{2}\right)$ can be taken uniformly in $z$ for $z$ in a bounded subset of $X$. Moreover, $f\left(x_{k}, u_{k}\right) \geq v\left(u_{k}\right) \geq 0$ and hence

$$
D_{u} f\left(x_{0}, u_{0}\right) r_{k}+\min _{z \in \Xi}\left\{D_{x} f\left(x_{0}, u_{0}\right) z+D^{2} f\left(x_{0}, u_{0}\right)((h, d),(h, d))\right\} \geq o(1),
$$

where $\Xi$ is the set formed by those $z \in X$ satisfying (7.7). By duality the minimum in (7.9) is equal to the maximum in (7.5) and hence

$$
D_{u} f\left(x_{0}, u_{0}\right) r_{k}+\operatorname{Min}_{h \in C\left(x_{0}\right)} \operatorname{Max}_{\lambda \in \Lambda_{0}\left(x_{0}\right)}\left\{D^{2} L\left(x_{0}, \lambda, u_{0}\right)((h, d),(h, d))-\sigma\left(\lambda, \mathcal{T}_{K}^{2}(h)\right)\right\} \geq o(1) .
$$

Since the above inequality holds for any $x_{0} \in \Delta_{1}(d)$, by the Slater condition and (7.4) it follows that $r_{k} \in T_{\Theta}^{2}\left(u_{0}, d\right)+o(1)$ which proves the second order regularity assertion.

\subsection{Differentiability of metric projections}

As an another application of Theorem 4.1, let us consider the question of directional differentiability of metric projections. Let $K$ be a convex closed subset of $\mathbb{R}^{n}$ and for a point $u \in \mathbb{R}^{n}$ denote by $P_{K}(u)$ the point in $K$ closest to $u$ (with respect to the Euclidean norm $\|\cdot\|)$. That is, $P_{K}(u)$ can be defined as the optimal solution of the problem

$$
\operatorname{Min}_{x \in K} \frac{1}{2}\|u-x\|^{2}
$$


and is called the metric projection of $u$ onto $K$. There are examples of a convex set $K$ in $\mathbb{R}^{3}$ (and even in $\mathbb{R}^{2}$ ) such that the corresponding metric projection is not directionally differentiable at a point $u_{0} \notin K([16,25])$.

The vector $u$ in (7.10) can be viewed as a perturbation parameter in a neighborhood of a given point $u_{0}$. Let us observe that all assumptions of Theorem 4.1 hold, provided the set $K$ is second order regular at the point $x_{0}:=P_{K}\left(u_{0}\right)$. For a given direction $d \in \mathbb{R}^{n}$, the auxiliary problem $\left(Q_{d}\right)$ can be written in the form

$$
\operatorname{Min}_{h \in C\left(x_{0}\right)}\left\{\|d-h\|^{2}-\sigma\left(\lambda, T_{K}^{2}\left(x_{0}, h\right)\right)\right\}
$$

where $\lambda=\left(u_{0}-x_{0}\right)$ and

$$
C\left(x_{0}\right)=\left\{h \in T_{K}\left(x_{0}\right):\left(u_{0}-x_{0}\right)^{T} h=0\right\} .
$$

By the discussion following Corollary 4.1, we have that the objective function in (7.11) is strongly convex. Since it is assumed that the set $K$ is second order regular at $x_{0}$, the second order tangent set $T_{K}^{2}\left(x_{0}, h\right)$ is non empty for every $h \in T_{K}\left(x_{0}\right)$. Moreover, since $T_{K}^{2}\left(x_{0}, h\right) \subset T_{T_{K}\left(x_{0}\right)}(h)$, we have that $\sigma\left(\lambda, T_{K}^{2}\left(x_{0}, h\right)\right)$ is finite valued for all $h \in C\left(x_{0}\right)$. It follows that the objective function in (7.11) is also finite valued for $h \in C\left(x_{0}\right)$, and hence the optimization problem (7.11) possesses a unique optimal solution. We obtain the following result (see Corollary 4.1).

Theorem 7.2 Suppose that the convex set $K \subset \mathbb{R}^{n}$ is second order regular at the point $x_{0}:=P_{K}\left(u_{0}\right)$. Then $P_{K}(u)$ is directionally differentiable at $u=u_{0}$ and $P_{K}^{\prime}\left(u_{0}, d\right)=h^{*}$, where $h^{*}$ is the optimal solution of the problem (7.11).

\subsection{An application to semi-definite programming}

Consider, for example, the space $\mathcal{S}^{p}$ of $p \times p$ symmetric matrices equipped with the scalar product $A \cdot B:=\operatorname{tr} A B$, where $A, B \in \mathcal{S}^{p}$, and the corresponding (Frobenius) norm $\|A\|:=$

$\sqrt{A \cdot A}=\sqrt{\sum_{i, j=1}^{p} a_{i j}^{2}}$. It is shown in [4] that the set $\mathcal{S}_{+}^{p}$ of positive semi-definite matrices is (inner) second order regular at every point. Therefore the theory presented in this paper can be applied in a straightforward manner to semi-definite programming problems.

Consider the cone $K:=\mathcal{S}_{+}^{p}$ and a point (matrix) $B \in \mathcal{S}_{+}^{p}$. If $B$ is positive definite, then $B$ belongs to the interior of $\mathcal{S}_{+}^{p}$ and in that case $T_{K}(B)=\mathcal{S}^{p}$. If $B$ is singular, then $T_{K}(B)=\left\{H \in \mathcal{S}^{p}: E^{T} H E \succeq 0\right\}$, where $E=\left[e_{1}, \ldots, e_{s}\right]$ is an $n \times s$ matrix whose columns $e_{1}, \ldots, e_{s}$ form an orthonormal basis of the null space of $B$. Furthemore, consider $H \in T_{K}(B)$. If the matrix $E^{T} H E$ is positive definite, then the second order tangent set $T_{K}^{2}(B, H)=\mathcal{S}^{p}$. If $E^{T} H E$ is singular, then

$$
T_{K}^{2}(B, H)=\left\{W \in \mathcal{S}^{p}: F^{T} E^{T} W E F \succeq 2 F^{T} E^{T} H B^{\dagger} H E F\right\} .
$$

where $B^{\dagger}$ is the Moore-Penrose pseudo inverse of $B$, and $F=\left[f_{1}, \ldots, f_{r}\right]$ with $f_{1}, \ldots, f_{r}$ being an orthonormal basis of the null space of the $s \times s$ matrix $E^{T} H E$ (see [26] for a discussion and derivation of these formulas). 
For example, consider the metric projection $P_{K}(\cdot)$ onto the set $K:=\mathcal{S}_{+}^{p}$. It is well known that $P_{K}(A)=\sum_{i \in I_{+}} \alpha_{i} e_{i} e_{i}^{T}$, where $A=\sum_{i=1}^{p} \alpha_{i} e_{i} e_{i}^{T}$ is the spectral decomposition of $A$ (i.e. $\alpha_{i}$ and $e_{i}$ are eigenvalues and corresponding orthonormal eigenvectors of $A$ ), $I_{+}:=\left\{i: \alpha_{i}>0\right\}, I_{-}:=\left\{i: \alpha_{i}<0\right\}$ and $I_{0}:=\left\{i: \alpha_{i}=0\right\}$. It follows from Theorem 7.2 that $P_{K}(\cdot)$ is directionally differentiable at every point $A \in \mathcal{S}^{p}$ even if $A$ has several zero eigenvalues. Suppose that $A \notin \mathcal{S}_{+}^{p}$. The directional derivative $P_{K}^{\prime}(A, D)$ is then given by the optimal solution of the corresponding auxiliary problem which we now calculate.

We have that the corresponding Lagrange multipler is given by $\lambda=\sum_{i \in I_{-}} \alpha_{i} e_{i} e_{i}^{T}$. Let $H \in C(B)$, where $B:=P_{K}(A)=\sum_{i \in I_{+}} \alpha_{i} e_{i} e_{i}^{T}$ and

$$
C(B)=\left\{Z \in T_{K}(B):\left(\sum_{i \in I_{-}} \alpha_{i} e_{i} e_{i}^{T}\right) \cdot Z=0\right\} .
$$

If $I_{+}$is empty, i.e. the matrix $A$ is negative semi-definite, then $B=P_{K}(A)=0$ and hence $\sigma\left(\lambda, T_{K}^{2}(B, H)=0\right.$. If $I_{+}$is non empty, then $B^{\dagger}=\sum_{i \in I_{+}} \alpha_{i}^{-1} e_{i} e_{i}^{T}$ and it follows from (7.13) that (cf. [26])

$$
\sigma\left(\lambda, T_{K}^{2}(B, H)\right)=2 \lambda \cdot\left(H B^{\dagger} H\right) .
$$

Therefore the auxiliary problem can be written in the form

$$
\operatorname{Min}_{H \in \mathcal{C}}\left\{\|D-H\|^{2}+\psi(H)\right\}
$$

where

$$
\psi(H)=\left\{\begin{array}{clc}
-2 \operatorname{tr}\left[\left(\sum_{i \in I_{-}} \alpha_{i} e_{i} e_{i}^{T}\right) H\left(\sum_{i \in I_{+}} \alpha_{i}^{-1} e_{i} e_{i}^{T}\right) H\right], & \text { if } & I_{+} \neq \emptyset \\
0, & \text { if } & I_{+}=\emptyset
\end{array}\right.
$$

and $\mathcal{C}=C(B)$ is the corresponding critical cone given by

$$
\mathcal{C}=\left\{Z \in \mathcal{S}^{p}: E^{T} Z E \succeq 0, \operatorname{tr} B Z=0\right\},
$$

where $E_{1}$ is the matrix whose columns are the vectors $e_{i}, i \in I_{-}, E_{2}$ is the matrix whose columns are $e_{i}, i \in I_{0}, E=\left[E_{1}, E_{2}\right]$, and $B:=\sum_{i \in I_{-}} \alpha_{i} e_{i} e_{i}^{T}$. Alternatively the cone $\mathcal{C}$ can be written in the form

$$
\mathcal{C}=\left\{Z \in \mathcal{S}^{p}: E_{1}^{T} Z E_{1}=0, E_{1}^{T} Z E_{2}=0, E_{2}^{T} Z E_{2} \succeq 0\right\} .
$$

We see that the objective function in (7.14) is quadratic and that $\mathcal{C}$ is a linear space iff the set $I_{0}$ is empty, i.e. iff $A$ does not have zero eigenvalues. Therefore we obtain that $P_{K}(\cdot)$ is differentiable at $A \notin K$ iff $A$ does not possess zero eigenvalues.

EXAMPLE. Consider the example discussed in the introduction. When $\alpha>0$ all calculations are simple, as computing second order terms is not necessary. When $\alpha=0$, Theorem 
5.1 applies. The critical cone is $C(\bar{x})=\left\{h \in \mathbb{R}^{2}: h_{1}=0 ; h_{2} \geq 0\right\}$, when $\lambda=\bar{\lambda}$, and the Lagrangian simplifies to $L(x, \bar{\lambda}, u)=\frac{1}{2} x_{2}^{2}$, The auxiliary problem $\left(\mathcal{D}_{d}^{2}\right)$ reduces to

$$
\operatorname{Min}_{h} h_{2}^{2}-\sigma\left(\lambda, \mathcal{T}_{\mathcal{S}_{+}}^{2}\left(0,\left(\begin{array}{cc}
h_{1} & 0 \\
0 & -h_{2}
\end{array}\right)\right)\right) \quad \text { s.t. } \quad h_{1}=0 ; h_{2} \geq 0 .
$$

The cost function of this subproblem is the sum of two nonnegative functions. Therefore it has the unique optimal solution $\hat{h}=0$, as $\mathcal{T}_{\mathcal{S}_{+}}^{2}(0,0)=\mathcal{S}_{+}$. It follows that any o(u)-optimal trajectory $x(u), u \geq 0$, satisfies $x(u)=o(\sqrt{u})$. As a matter of fact, by direct computation we find that the perturbed problem has a unique optimal solution $\bar{x}(u)=O\left(u^{2 / 3}\right)$.

\section{References}

[1] A. Auslender And R. Cominetti, First and second order sensitivity analysis of nonlinear programs under directional constraint qualification conditions, Optimization 21(1990), pp. 351-363.

[2] J.F. Bonnans, Directional derivatives of optimal solutions in smooth nonlinear programming, Journal of Optimization Theory and Applications, 73(1) (1992), pp. 27-45.

[3] J.F. Bonnans And R. Cominetti, Perturbed optimization in Banach spaces I: a general theory based on a weak directional constraint qualification, SIAM J. Control \& Opt. 34(4), (1996), pp. 1151-1171; Perturbed optimization in Banach spaces II: a theory based on a strong directional qualification, SIAM J. Control \& Opt. 34(4), (1996), pp. 1172-1189; Perturbed optimization in Banach spaces III: semi-infinite optimization, SIAM J. Control \& Opt. 34(6), (1996).

[4] J.F. Bonnans, R. Cominetti And A. Shapiro, Second order necessary and sufficient optimality conditions under abstract constraints, Rapport de Recherche INRIA 2952 (1996).

[5] J.F. Bonnans, A.D. Ioffe And A. Shapiro, Développement de solutions exactes et approchées en programmation non linéaire, Comptes Rendus de l'Académie des Sciences de Paris, t. 315 (1992), Série I, pp. 119-123.

[6] J.F. Bonnans And A. Shapiro, Optimization problems with perturbations, A guided tour, Rapport de Recherche INRIA 2872 (1996).

[7] S. Boyd, L. El Ghaoui, E. Feron and V. Balakrishnan, Linear Matrix inequalities. Studies in Applied Math. Vol. 15, SIAM, Philadelphia, PA, 1994.

[8] S. Boyd and L. Vandenberghe, Positive Definite Programming, SIAM Review, 38 (1996), pp. 49-95.

[9] R. CominetTi, Metric regularity, tangent sets and second order optimality conditions, Applied Math. \& Opt. 21 (1990), pp. 265-287.

$\mathrm{RR} \mathrm{n}^{\circ} 2989$ 
[10] R. Cominetti and J-P. Penot, Tangent sets to unilateral convex sets, Comptes Rendus de l'Académie des Sciences de Paris, tome 321 (1995), Série I, pp. 1631-1636.

[11] J. M. Danskin, The Theory of Max-Min and Its Applications to Weapons Allocation Problems, Springer, New York, 1967.

[12] J. Gauvin And R. Janin, Directional behavior of optimal solutions in nonlinear mathematical programming, Math. Oper. Res. 13(1988), pp. 629-649.

[13] A. Haraux, How to differentiate the projection on a convex set in Hilbert space. Some applications to variational inequalities, J. Math. Soc. Japan (29) (1977), pp. 615-631.

[14] A.D. Ioffe, On sensitivity analysis of nonlinear programs in Banach spaces : the approach via composite unconstrained optimization, SIAM J. Optimization 4(1994), pp. 1-43.

[15] H. KaWASAKI, An envelope-like effect of inifinitely many inequality constraints on second order necessary conditions for minimization problems, Math. Prog. 41 (1988), pp. 73-96.

[16] J. KRuskal, Two convex counterexamples: a discontinuous envelope function and a non-differentiable nearest point mapping, Proc. Amer. Math. Soc., 23 (1969), pp. 697703.

[17] S. Kurcyusz, On the existence and nonexistence of Lagrange multipliers in Banach spaces, Journal of Optimization Theory and Applications, 20 (1976), pp. 81-110.

[18] F. Mignot, Contrôle dans les inéquations variationnelles elliptiques, J. Funct. Anal. 22 (1976), 25-39.

[19] S. M. Robinson, First order conditions for general nonlinear optimization, SIAM Journal on Applied Mathematics, 30 (1976), pp. 597-607.

[20] S. M. Robinson, Stability theorems for systems of inequalities, Part II: differentiable nonlinear systems, SIAM J. Numerical Analysis, 13 (1976), pp. 497-513.

[21] R. T. Rockafellar, Conjugate Duality and Optimization, Regional Conference Series in Applied Mathematics, SIAM, Philadelphia, 1974.

[22] A. Shapiro, Perturbation theory of nonlinear programs when the set of optimal solutions is not a singleton, Applied Mathematics \& Optimization 18 (1988), pp. 215-229.

[23] A. ShapIRo, Sensitivity analysis of nonlinear programs and differentiability properties properties of metric projections, SIAM J. Control \& Opt. 26(1988), pp. 628-645.

[24] A. Shapiro, On Lipschitzian stability of optimal solutions of parametrized semi-infinite programs, Math. Oper. Research 19 (1994), pp. 743-752.

INRIA 
[25] A. ShapIRo, Directionally nondifferentiable metric projection, Journal of Optimization Theory and Applications, 81(1994), pp. 203-204.

[26] A. ShapIRo, First and second order analysis of nonlinear semidefinite programs, Mathematical Programming, Series B, to appear.

[27] A. Shapiro And J. F. Bonnans, Sensitivity analysis of parametrized programs under cone constraints, SIAM J. Control and Optimization, 30 (1992), pp. 1409-1422.

[28] J. Zowe AND S. KuRCYusz, Regularity and stability for the mathematical programming problem in Banach spaces, Applied Mathematics and Optimization, 5 (1979), pp. 49-62.

$\mathrm{RR} \quad \mathrm{n}^{\circ} 2989$ 
Unité de recherche INRIA Lorraine, Technopôle de Nancy-Brabois, Campus scientifique, 615 rue du Jardin Botanique, BP 101, 54600 VILLERS LÈS NANCY

Unité de recherche INRIA Rennes, Irisa, Campus universitaire de Beaulieu, 35042 RENNES Cedex

Unité de recherche INRIA Rhône-Alpes, 655, avenue de l'Europe, 38330 MONTBONNOT ST MARTIN

Unité de recherche INRIA Rocquencourt, Domaine de Voluceau, Rocquencourt, BP 105, 78153 LE CHESNAY Cedex

Unité de recherche INRIA Sophia-Antipolis, 2004 route des Lucioles, BP 93, 06902 SOPHIA-ANTIPOLIS Cedex

Éditeur
INRIA, Domaine de Voluceau, Rocquencourt, BP 105, 78153 LE CHESNAY Cedex (France)

ISSN 0249-6399 